15

\title{
Дисперсия объемных волн в структуре „графен-диэлектрик-графен“
}

\author{
(ㄱ А.С. Абрамов, Д.А. Евсеев, И.О. Золотовский, Д.И. Семенцов \\ Ульяновский государственный университет, \\ 432970 Ульяновск, Россия \\ e-mail: aleksei_abramov@mail.ru
}

Поступила в редакцию 15.08.2018 г.

В окончательной редакции 21.09.2018 г.

Исследованы дисперсионные свойства первых волноводных мод в диэлектрической пленке с обкладками из графена при различных значениях его химического потенциала. Рассмотрено управление фазовой и групповой скоростью первой волноводной моды. Обнаружены спектральные интервалы, в которых наблюдается малая фазовая и отрицательная групповая скорости волноводных мод. Показано, что их дисперсионные характеристики могут перестраиваться с помощью внешнего электрического поля.

DOI: $10.21883 /$ OS.2019.02.47209.239-18

\section{Введение}

В последнее время внимание исследователей привлекают металло-диэлектрические планарные структуры, в частности их волноводные свойства [1-3]. К одному из таких важных свойств можно отнести наличие у металлов широкой частотной области, в которой диэлектрическая проницаемость отрицательна, что позволяет возбуждать на границах раздела металла с диэлектриками поверхностные плазмон-поляритоны (ПП) [4]. Волновые характеристики поверхностных и объемных ПП во многом определяются характером дисперсии материальных параметров граничащих сред. Поведение ПП в металлодиэлектрических волноводных структурах и возможность их практического применения достаточно подробно рассматривались в работах [3,5-7]. Однако использование в качестве волноведущего слоя металлических пленок неизбежно приводит к существенным потерям и значительному уменьшению длины пробега ПП.

Значительный интерес представляют волноведущие структуры, в которых возможно существенное замедление распространяющихся волн, что важно для решения многих прикладных задач фотоники. Существует достаточно большое количество материалов и структур, в которых теоретически предсказано и экспериментально реализовано распространение медленных волн. Прежде всего это высокотемпературные сверхпроводники [8], фотонные кристаллы и метаматериалы [9], планарные структуры, содержащие полупроводниковые пленки [10]. Замедление и управление дисперсионными характеристиками волн в направляющих структурах может быть достигнуто также в результате использования при их формировании слоев графена, химический потенциал (ХП) которого существенно зависит от температуры и внешнего электрического поля $[11,12]$. Слои графена могут использоваться как покрытие диэлектрической сердцевины в планарных волноводах $[11,13-15]$ или в волоконных световодах [16], а также в более сложных структурах. Возможность управления дисперсионными свойствами волноводных ТЕ-мод в прямоугольном волноводе с металлическими стенками, диэлектрическим заполнением и слоем графена за счет изменения не только ХП, но и положения графенового слоя внутри волновода, показана в работе [17]. В работе [18] исследованы дисперсионные особенности поверхностных плазмонов в структуре „прямоугольный диэлектрический волновод-монослой графена-покровная среда“. Эффективное управление свойствами направляемых волн в различных волноводных структурах на основе графена может осуществляться как электрическим, так и магнитным полями [19].

В настоящей работе исследуется влияние на дисперсионные свойства волноводных мод в диэлектрической пленке слоев графена, нанесенных на ее поверхности. Рассматривается возможность управления фазовой и групповой скоростями волноводных мод за счет симметричного и несимметричного изменений ХП графеновых слоев.

\section{Материальные параметры структуры}

Исследовать режим волноводного распространения мод будем в планарной структуре, состоящей из тонкой диэлектрической пленки толщиной $d$, диэлектрическая проницаемость (ДП) которой $\varepsilon_{d}$ в рассматриваемом спектральном диапазоне не обладает дисперсией и является действительной величиной. На поверхности пленки $z=0$ и $z=d$ нанесены два монослоя графена с ХП $\mu_{1}$ и $\mu_{2}$, следовательно, с различными проводимостями $\sigma_{1}, \sigma_{2}$. Пленка со слоями графена находится между двух немагнитных сред с ДП $\varepsilon_{1}$ и $\varepsilon_{2}$. Геометрия структуры представлена на рис. 1.

Частотная зависимость действительной и мнимой частей комплексной поверхностной проводимости $\sigma=\sigma^{\prime}+i \sigma^{\prime \prime}$ допированного графена определялась в 


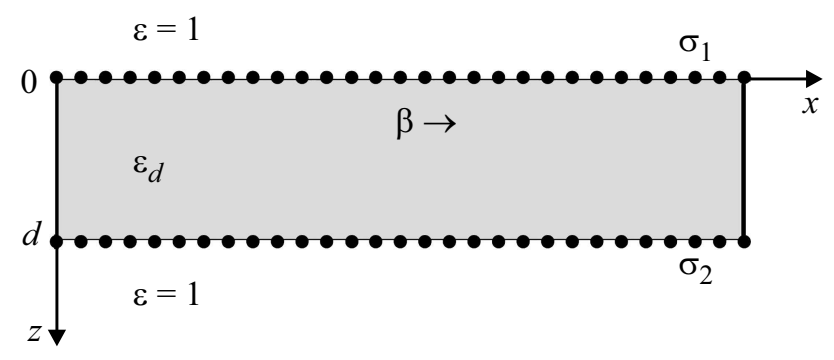

Рис. 1. Геометрия структуры.

рамках модели Кубо соотношениями [20-22]:

$$
\begin{gathered}
\frac{\sigma^{\prime}}{\sigma_{0}}=\frac{1}{2}+\frac{1}{\pi} \operatorname{arctg}\left(\frac{h \omega-2 \mu}{2 k_{b} T}\right), \\
\frac{\sigma^{\prime \prime}}{\sigma_{0}}=\frac{1}{2 \pi}=\left[\frac{16 k_{B} T}{h \omega} \ln \left(2 \cosh \left(\frac{\mu}{2 k_{B} T}\right)\right)\right. \\
\left.-\ln \left(\frac{(h \omega+2 \mu)^{2}}{(h \omega-2 \mu)^{2}+\left(2 k_{B} T\right)^{2}}\right)\right] .
\end{gathered}
$$

Здесь $\sigma_{0}=e^{2} / 4 h-$ фундаментальная (статическая) проводимость графена, $e-$ заряд электрона, $h-$ постоянная Планка, $\omega-$ частота, $k_{B}-$ постоянная Больцмана, $T$ - температура, ХП $\mu=\hbar v_{F} \sqrt{\pi n_{0}}$, где $n_{0}$ и $v_{0}$ - концентрация носителей заряда и скорость Ферми в графене.

На рис. 2 представлены частотные зависимости действительной и мнимой частей (сплошные и пунктирные кривые) поверхностной проводимости графена, построенные в соответствии с выражениями (1) для значений ХП $\mu=(0.1,0.0,0.1,0.2,0.3) \mathrm{eV}$ (кривые $1-5)$, который можно изменять в эксперименте с помощью внешнего электрического поля. Здесь и далее численный анализ проводится при рабочей температуре $T=300 \mathrm{~K}$. Нулевые и отрицательные значения ХП достигаются за счет приложения нулевого и обратного напряжений смещения соответственно [23]. Видно, что с ростом ХП область роста собственной проводимости $\sigma^{\prime}$ смещается в область более высоких частот (сплошные кривые 3-5). Такое же смещение испытывает минимум мнимой части проводимости $\sigma^{\prime \prime}$ (штриховые кривые 3-5). При нулевом и отрицательных значениях ХП действительная часть проводимости практически не зависит от частоты (сплошные кривые 1-2), а мнимые части при этом не заходят в отрицательную область (сплошные кривые 1-2). При наличии отрицательных значений мнимой части проводимости в достаточно широкой частотной области (кривые 3-5) в диэлектрическом волноводе с графеновыми слоями на поверхностях возможно распространение как волноводных, так и поверхностных мод. При условии $\sigma^{\prime \prime}>0$ возможно распространение только объемных волн. Данное условие выполняется для всех значений ХП (кривые 1-5).
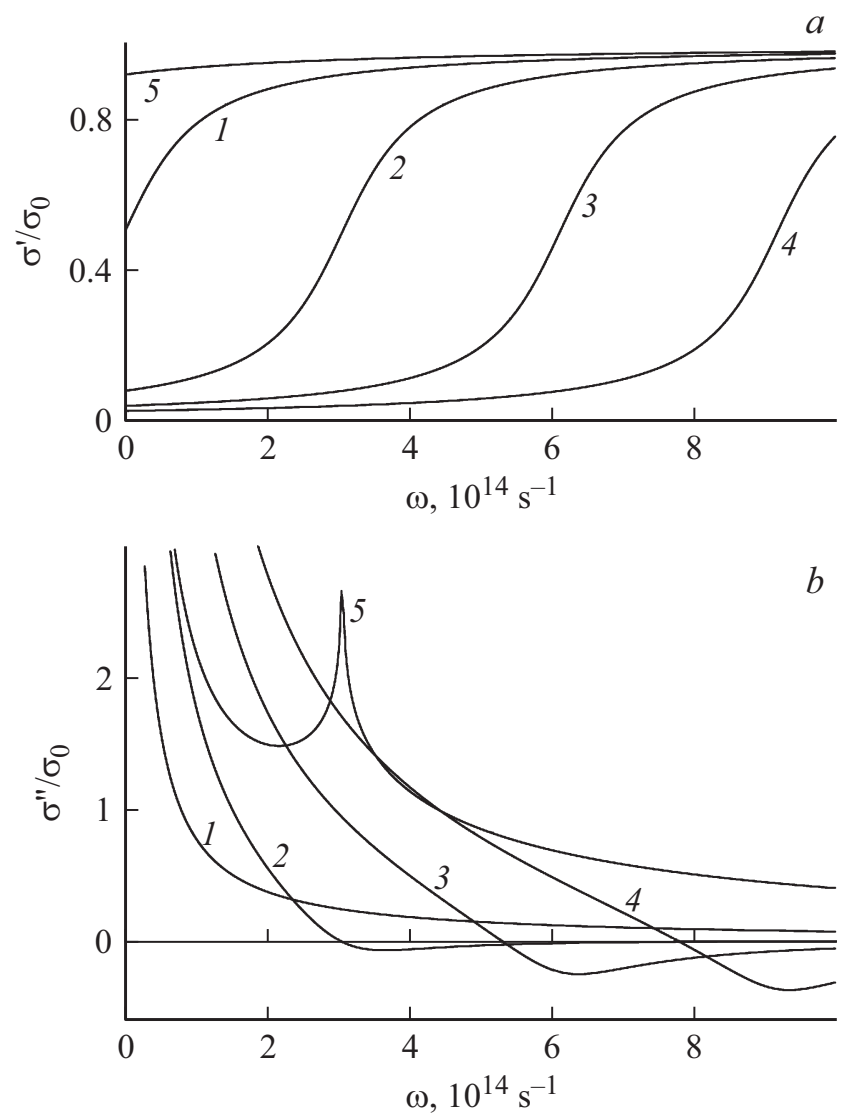

Рис. 2. Частотные зависимости действительной $\sigma^{\prime}(a)$ и мнимой части $\sigma^{\prime \prime}(b)$ поверхностной проводимости графена для $\mu=0,0.1,0.2,0.3,-0.1 \mathrm{eV}$ (кривые $1-5$ ).

\section{Волновые поля и дисперсионное соотношение}

В исследуемой волноводной структуре могут распространяться оба типа мод линейной поляризации - ТМ и ТЕ. Компоненты волнового поля этих мод зависят от времени и координат следующим образом:

$$
F_{\alpha}(x, z, t)=F_{\alpha}(z) \exp [i(\omega t-\beta x)]
$$

где $F_{\alpha}(z)-$ профильные функции, $\omega-$ частота, $\beta-$ комплексная константа распространения. Для волны ТМ-типа связь между компонентами поля в каждой из сред $(j=1,2,3)$ определяется уравнениями:

$$
\begin{aligned}
\frac{\partial^{2} H}{\partial z^{2}}-q_{j}^{2} H_{y} & =0, \quad E_{x}=\frac{i}{k_{0} \varepsilon_{j}} \frac{\partial H_{y}}{\partial z}, \\
E_{z} & =-\frac{\beta}{k_{0} \varepsilon_{j}} H_{y},
\end{aligned}
$$

где $q_{j}-$ поперечные компоненты волнового вектора в каждой из сред. При этом $q_{1,3}^{2}=\beta^{2}-k_{0}^{2} \varepsilon_{1,3}$, $q_{2}^{2}=k_{0}^{2} \varepsilon_{d}-\beta^{2}$, где $k_{0}=\omega / c, c-$ скорость света в вакууме. 
Для решения граничной задачи используем граничные условия для тангенциальных компонент электрического и магнитного полей моды:

$$
\begin{aligned}
& E_{1 x}=E_{2 x}, \quad H_{1 y}-H_{2 y}=\frac{4 \pi}{c} \sigma_{1} E_{1 x} \quad(z=0), \\
& E_{2 x}=E_{3 x}, \quad H_{2 y}-H_{3 y}=\frac{4 \pi}{c} \sigma_{2} E_{2 x} \quad(z=d) .
\end{aligned}
$$

Приравнивая детерминант системы двух пар уравнений (4) к нулю, получаем дисперсионное соотношение для распространяющихся в структуре волн:

$$
\begin{aligned}
q_{d} d= & \pi m-\operatorname{arctg}\left(\frac{\varepsilon_{1} q_{d}}{q_{1} \varepsilon_{d}}\left(1-\frac{4 \pi \sigma_{1}}{c} \frac{q_{1}}{i k_{0} \varepsilon_{1}}\right)\right) \\
& -\operatorname{arctg}\left(\frac{\varepsilon_{3} q_{d}}{q_{3} \varepsilon_{d}}\left(1-\frac{4 \pi \sigma_{2}}{c} \frac{q_{3}}{i k_{0} \varepsilon_{3}}\right)\right) .
\end{aligned}
$$

Это уравнение с учетом комплексности входящих в него параметров определяет связь действительной и мнимой частей волнового числа $\beta=\beta^{\prime}-i \beta^{\prime \prime}$ с частотой электромагнитной волны. Здесь уравнение (5) записано для случая различных проводимостей на границах волновода и содержит три различные поперечные компоненты волнового вектора. В приближении $\sigma_{1}=\sigma_{2}=0$ (т. е. в отсутствие слоев графена) выражение (5) сводится к стандартному дисперсионному соотношению для объемных мод в диэлектрическом волноводе [24].

\section{Численный анализ}

Ниже приведены результаты численного анализа режимов распространения волноводных волн в рассматриваемой структуре. Далее будем считать, что структура находится в вакууме, поэтому $\varepsilon_{1}=\varepsilon_{3}=1$. На следующих двух рисунках приведены дисперсионные зависимости для волноводных мод в рассматриваемой структуре, которые являются решением уравнения (5) и представлены как зависимость частоты от нормированной действительной части константы распространения $\beta^{\prime}$. Нормировка проводилась на величину $k_{T}=k_{B} T / h c$, значение которой для рабочей температуры $T=300 \mathrm{~K}$ составляет $k_{T}=1314.24 \mathrm{~cm}^{-1}$. На рис. $3, a$ указанные зависимости получены для первых трех волноводных мод с номерами $m=0,1,2$ (кривые $1-3$ ) при одинаковых значениях ХП графеновых слоев на обеих границах $\mu_{1}=\mu_{2}=(0.1,0.2) \mathrm{eV}$ (сплошные и штриховые кривые). Видно, что при выбранном фиксированном значении постоянной распространения изменение ХП слоев графена с $0.2 \mathrm{eV}$ до $0.1 \mathrm{eV}$ позволяет существенно расширить спектральный диапазон существования волноводных мод в структуре. Так, для первой волноводной моды при значении $\beta^{\prime} / k_{T}=400$ подобное изменение ХП приводит к расширению спектрального интервала более чем в два раза. На рис. $3, b$ дисперсионные зависимости приведены для толщины слоя диэлектрика (кривые $1-3$ ). Видно, что с увеличением толщины пленки диэлектрика
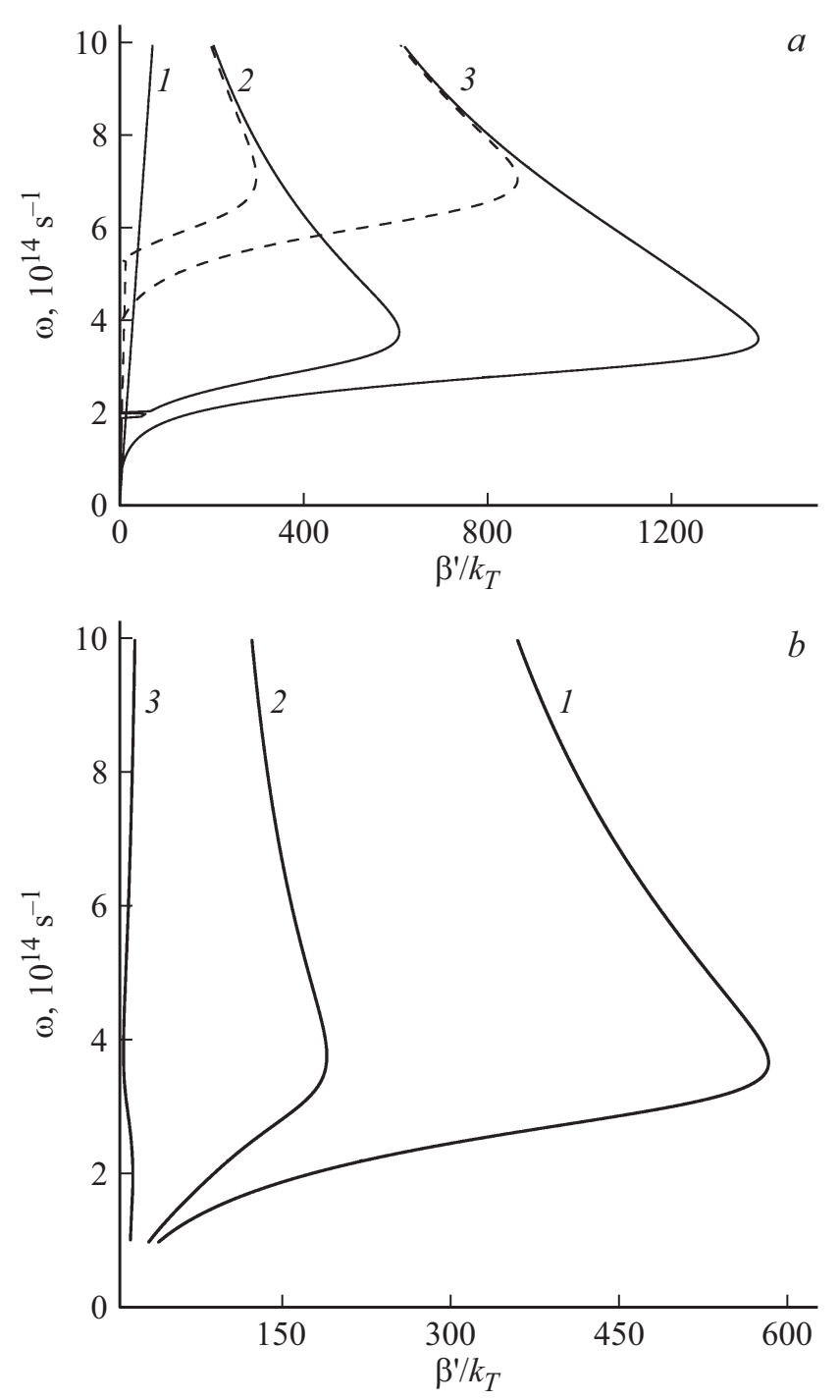

Рис. 3. Дисперсионные зависимости для волноводных мод с $m=0,1,2$ (a, кривые 1-3) при $\mu_{1}=\mu_{2}=0.1,0.2 \mathrm{eV}$ (сплошные и штриховые кривые), для первой моды при $\mu_{1}=\mu_{2}=0.1 \mathrm{eV}$ и $d=10,20,50 \mathrm{~nm}(b$, кривые $1-3)$.

мода по-прежнему существует во всем рассматриваемом спектральном интервале, но диапазон постоянных распространения, соответствующих фиксированным частотам, существенно сужается. Уже при толщине пленки $d=10,20,50 \mathrm{~nm}$ (кривая 3) закон дисперсии становится практически линейным.

В работе [17] управление дисперсионными свойствами первых ТЕ-мод в прямоугольном волноводе с металлическими стенками, диэлектрическим заполнением и слоем графена также осуществлялось за счет изменения ХП графена. Было также установлено влияние ХП на коэффициенты пропускания и отражения волноводных мод. При этом дополнительное влияние на дисперсию волны и волноводные характеристики оказывает расстояние от монослоя графена до металлических стенок волновода. 

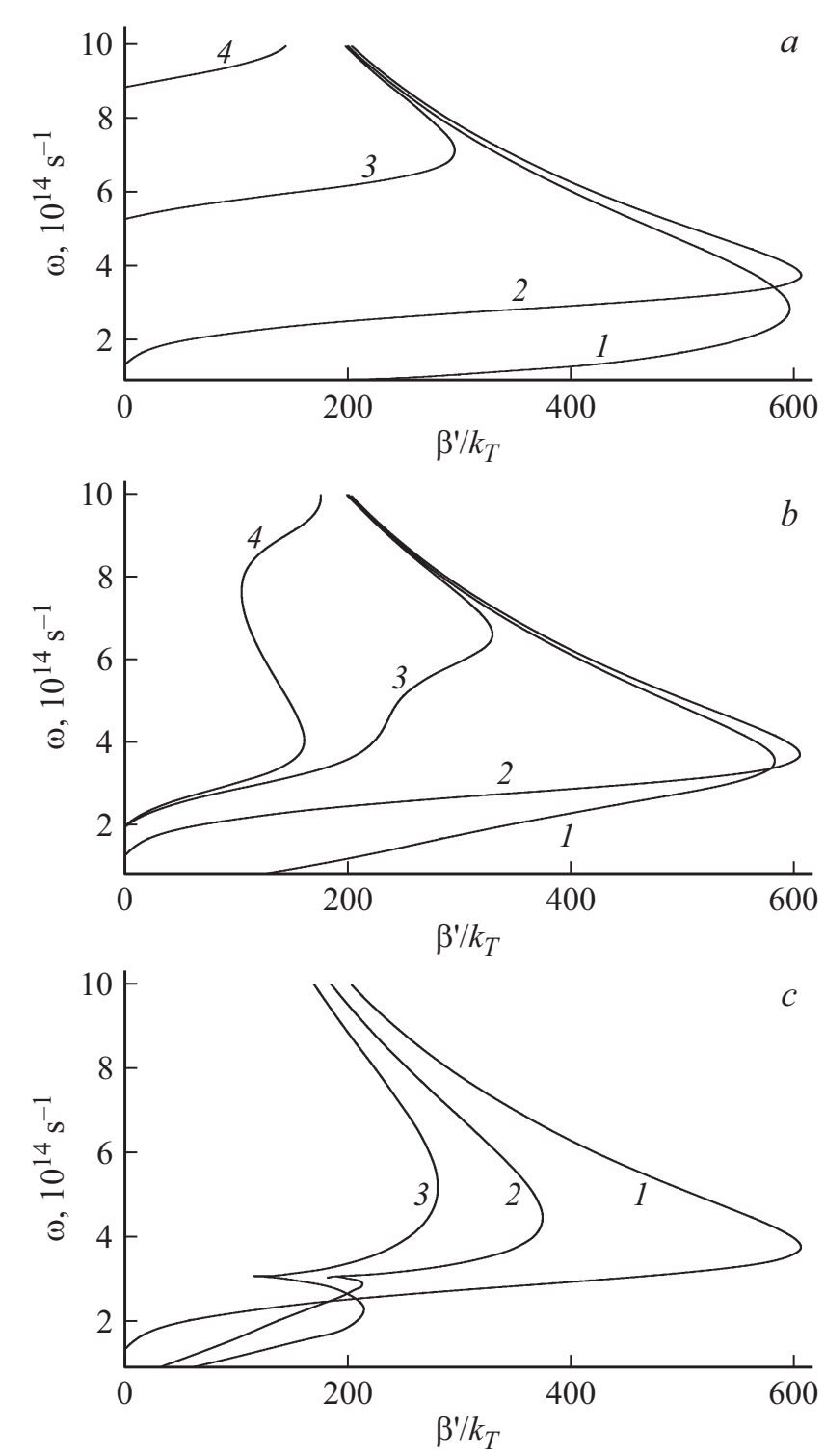

Рис. 4. Дисперсионные зависимости для первой волноводной моды в структуре с $\mu_{1}=\mu_{2}=0,0.1,0.2,0.3 \mathrm{eV}$ ( $a$, кривые $1-4) ; \mu_{1}=0.1 \mathrm{eV}$ и $\mu_{2}=0.0,0.1,0.2,0.3 \mathrm{eV}$ (b, кривые $1-4) ; \mu_{1}=0.1 \mathrm{eV}, \mu_{2}=0.1,-0.1 \mathrm{eV}$ (c, кривые 1,2$)$ и $\mu_{1}=\mu_{2}=-0.1 \mathrm{eV}$ (c, кривая 3$)$.

На рис. 4 для первой волноводной моды приведены дисперсионные зависимости для различных комбинаций ХП графеновых слоев. Вначале рассмотрим симметричную ситуацию, когда $\mu_{1}=\mu_{2}=0.0,0.1$, $0.2,0.3 \mathrm{eV}$ (рис. 4, $a$, кривые 1-4). Анализ показывает, что в рассматриваемом спектральном диапазоне $\omega=\left(10^{14} \ldots 10^{15}\right) \mathrm{c}^{-1}$ первая мода может распространяться в структуре, только если $\mu_{1}=\mu_{2} \leq 0.3 \mathrm{eV}$. При этом максимальное значение постоянной распространения $\beta^{\prime} \approx 600 k_{T}$ для $\mu_{1}=\mu_{2}=0.1 \mathrm{eV}$, что отвечает наименьшему значению длины пробега волны при выбранных значениях параметров. При увеличении ХП графеновых обкладок область существования первой моды смещается к более высоким частотам, а диапазон возможных постоянных распространения сужается, т.е. сужается интервал длин пробега моды при фиксированном значении частоты. Так, при значениях $\mu_{1}=\mu_{2}=0.5 \mathrm{eV}$ волноводные моды могут существовать в области частот $\omega=(1.6 \ldots 2.0) \cdot 10^{15} \mathrm{~s}^{-1}$. При этом их максимальное значение постоянной распространения снижается до $\beta^{\prime} / k_{T} \approx 23$, что отвечает увеличению длины пробега волны при выбранных значениях параметров.

В несимметричной ситуации, когда ХП изменяется только для одного из слоев графена, например $\mu_{1}=0.1 \mathrm{eV}$, а $\mu_{2}=0.0,0.2,0.3 \mathrm{eV}$ (рис. 4, $b$, кривые 1 , $3,4)$, дисперсионные зависимости имеют более сложный вид. Видно, что чем больше разница ХП слоев, тем в большей степени меняется характер дисперсии волноводных мод и начинают проявляться особенности, связанные с наличием графена: в частности, область их существования также сужается, но появляются дополнительные экстремумы зависимости $\beta^{\prime}(\omega)$, соответствующие бесконечно большой групповой скорости. Более того, варьируя значения ХП слоев графена, можно смещать частотный диапазон, в котором групповая скорость волн отрицательна. При отрицательных значениях ХП на одной из обкладок на дисперсионных зависимостях проявляются изгибы, обусловленные резонансом мнимой части проводимости графена: $\mu_{1}=0.1 \mathrm{eV}$, $\mu_{2}=0.1,-0.1 \mathrm{eV}$ (рис. 4,c, кривые 1,2). Эффект проявляется сильнее в случае, когда значения ХП обоих слоев графена отрицательны: $\mu_{1}=\mu_{2}=-0.1 \mathrm{eV}$ (кривая 3).

На рис. 5 приведены распределения электрического и магнитного полей первой моды $(m=1)$ по сечению структуры, отвечающие частоте $\omega=4 \cdot 10^{14} \mathrm{~s}^{-1}$ и различным значениям ХП $\mu_{1}$ и $\mu_{2}$. Распределения построены для двух случаев, когда $\mu_{1}=\mu_{2}=-0.1,0.0,0.1 \mathrm{eV}$ (соответственно штриховые, пунктирные и сплошные кривые на рис. $5, a)$ и $\mu_{1}=(0,0.1,0.3) \mathrm{eV}, \mu_{2}=0.1 \mathrm{eV}$ (штриховые, сплошные и пунктирные кривые на рис. $5, b)$. Видно, что наличие монослоев графена приводит к разрыву магнитного поля на границах волноводного слоя. При симметричных значениях ХП распределение полей в волноводной структуре также обладает симметрией. На рисунке приведен случай антисимметричного электрического поля и симметричного магнитного поля. При $\mu_{1} \neq \mu_{2}$ симметрия в распределении полей пропадает. Изменяя величину ХП, можно существенно модифицировать распределение волнового поля в структуре и тем самым влиять на ее дисперсионные свойства.

Далее на основании приведенных дисперсионных зависимостей было рассмотрено влияние графеновых обкладок на частотные зависимости фазовых (рис. 6) и групповых (рис. 7) скоростей для первой волноводной моды. Для построения указанных зависимостей использовались следующие значения ХП: $\mu_{1}=\mu_{2}=-0.1,0.1,0.2,0.3 \mathrm{eV}$ (рис. 6 и 7, $a$, кривые 1 4) и $\mu_{1}=0.1 \mathrm{eV}, \mu_{2}=-0.1,0.1,0.2,0.3 \mathrm{eV}$ (рис. 6 и 7, $b$, 

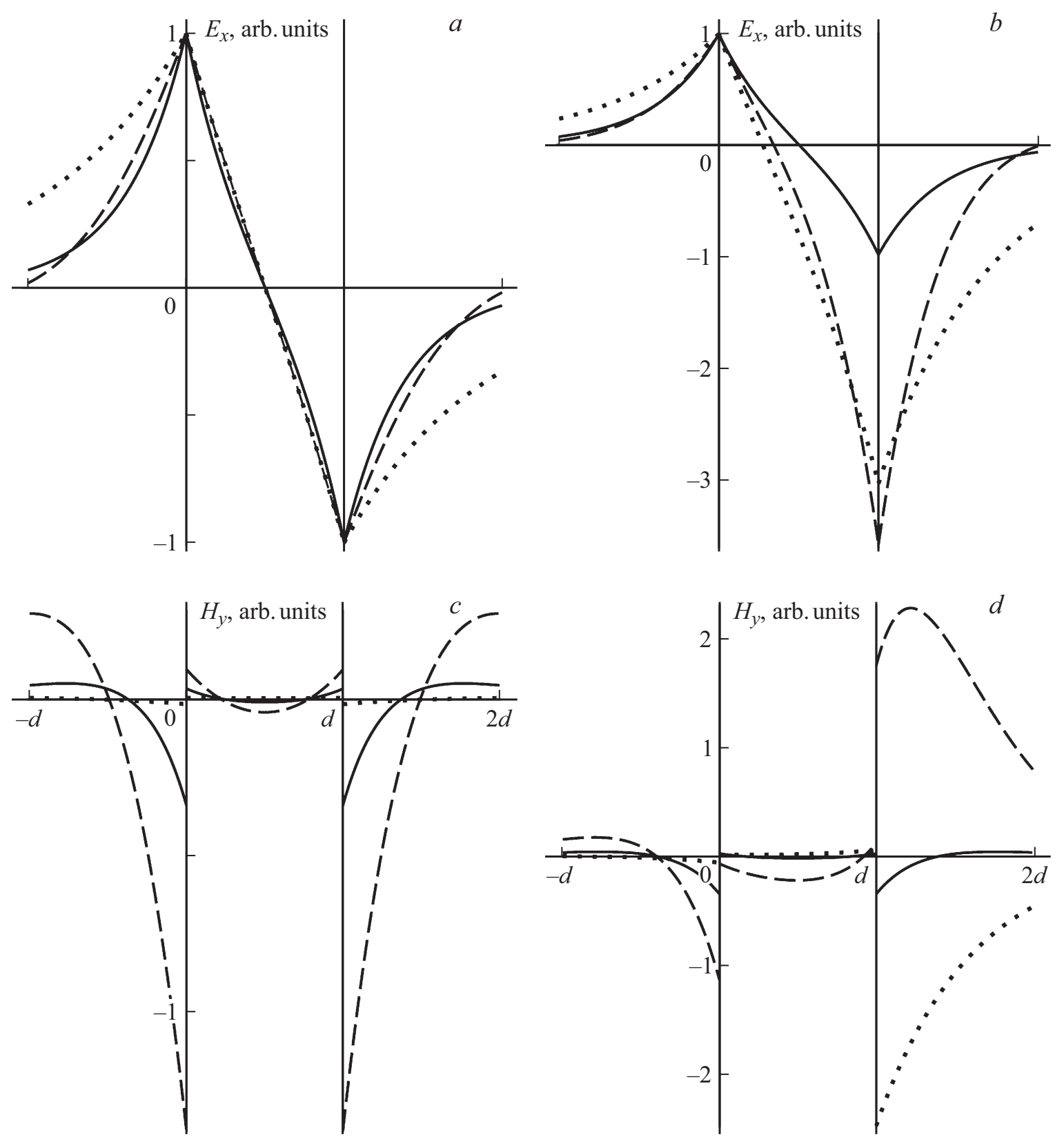

Рис. 5. Распределение электрического и магнитного полей волны по сечению структуры для первой волноводной моды $\mu_{1}=\mu_{2}=-0.1,0.0,0.1 \mathrm{eV}$ ( $a$, штриховые, пунктирные и сплошные кривые) и $\mu_{1}=0,0.1,0.3 \mathrm{eV}, \mu_{2}=0.1 \mathrm{eV}(b$, штриховые, сплошные и пунктирные кривые), $\omega=6 \cdot 10^{14} \mathrm{~s}^{-1}$.

кривые 1-4). Для фазовой скорости $v_{p h}=\omega / \beta^{\prime}$ после резкого спада в узком спектральном интервале наблюдается дальнейший плавный рост, характерный для значений ХП $\mu_{1}=\mu_{2}=0.1,0.2 \mathrm{eV}$ (рис. 6, a, кривые 2, 3). Минимальные значения, которых может достигать фазовая скорость волны в структуре $v_{p h} / c ; 0.2$ (т. е. замедление в 50 раз), соответствуют нулевому ХП (кривая 1). C точки зрения замедления волны, оптимальным является выбор нулевого ХП, при котором $v_{p h}$ можно считать малой и постоянной величиной в диапазоне $\omega=(1 \ldots 3) \cdot 10^{14} \mathrm{~s}^{-1}$. Для случая разных ХП на гра- ницах волновода функция $v_{p h}(\omega)$ может иметь один или несколько экстремумов (рис. $6, b$, кривые 3,4 ), что открывает дополнительные возможности для управления. Отметим, что по сравнению со случаем одинаковых ХП обкладок, когда области наблюдения волн сужаются с одновременным увеличением ХП, при фиксированном ХП верхней границы и изменении ХП нижней сужения области существования волн не происходит (рис. $6, b$, кривые 2-4).

Замедление первых волноводных мод в структуре „диэлектрический волновод-монослой графена-покровная 
среда“ установлено также в работе [18]. Так, в среднем ИК диапазоне $(8-16 \mu \mathrm{m})$ значения модового эффективного показателя преломления достигало значений, близких к 100. Это указывает на то, что фазовые скорости распространяющихся в структуре волн могут быть практически на 2 порядка меньше скорости света в вакууме.

Для групповой скорости $v_{g}=d \omega / d \beta^{\prime}$ до критической частоты, отвечающей перегибу дисперсионной кривой (и, как следствие, стремлению $\left.v_{g} \rightarrow \infty\right)$, характерны положительные значения. Для волн с частотами $\omega>\omega_{c r}$ групповая скорость отрицательна. Частотная область, в которой групповая скорость отрицательна, может быть смещена за счет изменения ХП нижней обкладки (рис. 7, $a$, кривые 1-4). При значениях ХП $\mu_{1}=0.1 \mathrm{eV}, \mu_{2}=0.2 \mathrm{eV}$ групповая скорость принимает малые и положительные значения в частотном диапазоне $\omega=(1.0 \ldots 3.5) \cdot 10^{14} \mathrm{~s}^{-1}$ (рис. 7, $b$, кривая 3). Для отрицательных значений ХП появляется дополнительная частотная область, в которой наблюдается отрицательная групповая скорость волноводной моды (кривые 2, 3).
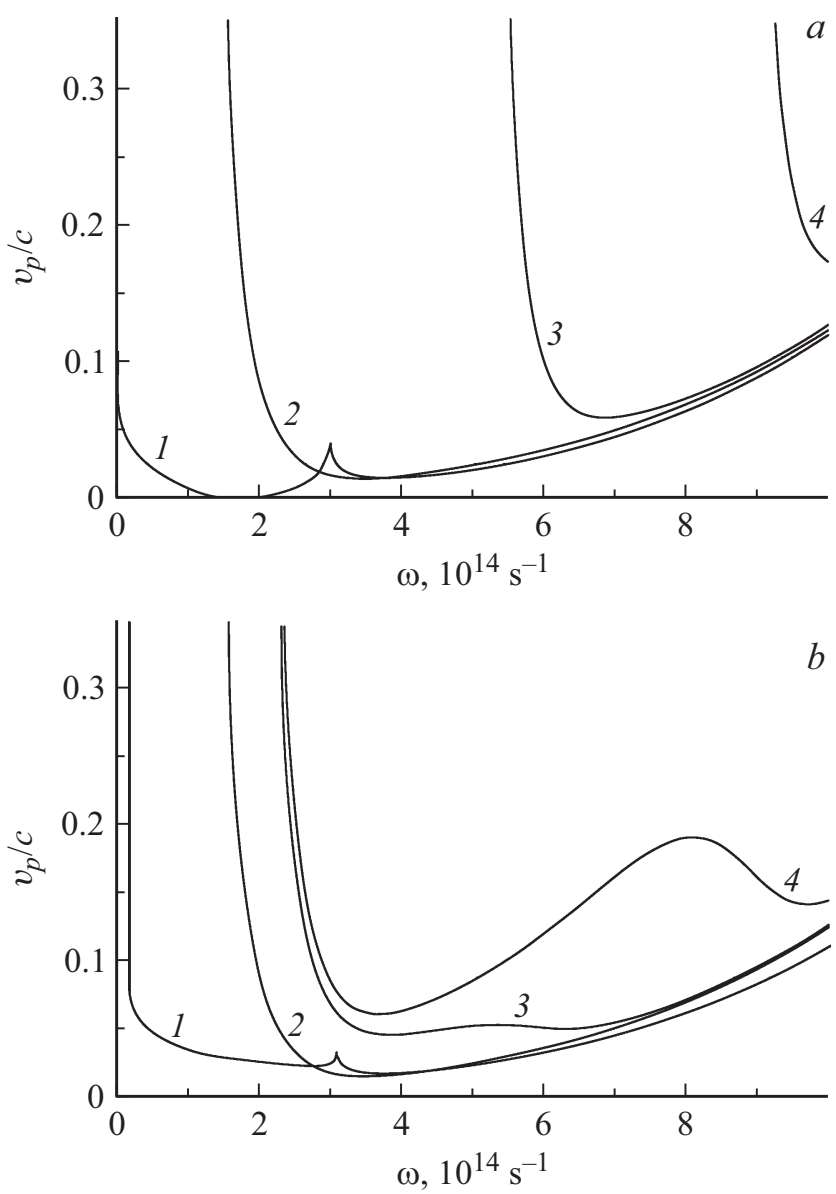

Рис. 6. Частотные зависимости фазовых скоростей для первой волноводной моды при $\mu_{1}=\mu_{2}=-0.1,0.1,0.2,0.3 \mathrm{eV}$ $(a$, кривые $1-4)$ и $\mu_{1}=0.1 \mathrm{eV}, \mu_{2}=-0.1,0.1,0.2,0.3 \mathrm{eV}(b$, кривые 1-4).
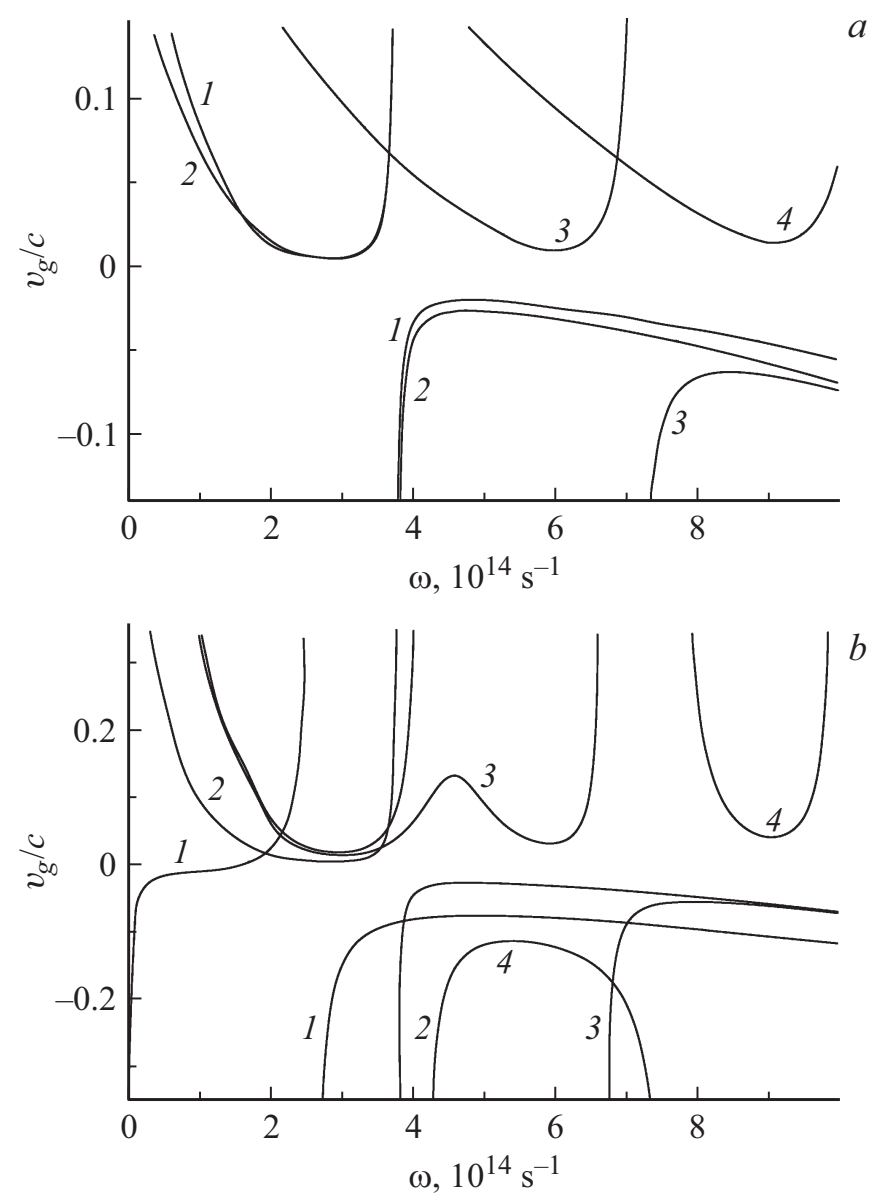

Рис. 7. Частотные зависимости групповых скоростей для первой волноводной моды при $\mu_{1}=\mu_{2}=-0.1,0.1,0.2,0.3 \mathrm{eV}$ $(a$, кривые $1-4)$ и $\mu_{1}=0.1 \mathrm{eV}, \mu_{2}=-0.1,0.1,0.2,0.3 \mathrm{eV}(b$, кривые 1-4).

\section{Заключение}

В настоящей работе рассмотрены особенности распространения первых волноводных мод в диэлектрическом слое, находящемся между двумя монослоями графена. Изменение ХП графена может осуществляться в результате изменения напряжения затвора между графеновыми обкладками. Для первой волноводной моды показана возможность управления ее дисперсионными параметрами за счет изменения ХП графеновых слоев. В частности, можно управлять областью существования волноводной моды, ее фазовой и групповой скоростью. Рассматриваемая волноводная структура может быть положена в основу компактных диэлектрических волноведущих структур, дисперсионные характеристики которых могут быть перестроены внешним электрическим полем в процессе распространения мод.

Работа выполнена при поддержке Российского Научного Фонда (в рамках проекта № 17-72-10135) и министерства образования и науки РФ (проект № 14.Z50.31.0015). 


\section{Список литературы}

[1] Iorsh I.V., Shadrivov I.V., Belov P.A., Kivshar Yu.S. // JETP Letters. 2013. V. 97. N 5. P. 287. doi 10.7868/S0370274X13050020

[2] Федянин Д.Ю., Арсенин А.В., Лейман В.Г., Гладун А.Д. // Квант. Электрон. 2009. T. 39. № 8. C. 745; Fedyanin D.Yu., Arsenin A.V., Leiman V.G., Gladun A.D. // Quantum Electronics. 2009. V. 39. N 8. P. 745. doi 10.1070/QE2009v039n08ABEH014072

[3] Suarez I., Fernando A., Marques-Hueso J., Diez A., Abarguez R., Rodriguez-Canto P., Martinez-Pastor J. // Nanophotonics. 2017. V. 6. N 5. P. 1109. doi 10.1515/nanoph2016-0166

[4] Агранович В.М., Миллс Д.Л. Поверхностные поляритоны: электромагнитные волны на поверхностях и границах раздела сред. М.: Наука, 1985. 525 c.; Agranovich V.M., Mills D.L. Surface Polaritons - Electromagnetic waves at surfaces and interfaces. Elsevier Science Ltd, 1982. 734 p.

[5] Fong N.R., Menotti M., Lisicka-Skrzek E., Northfield H., Olivieri A., Tait N., Liscidini M., Berini P. // ACS Photonics. 2017. V. 4. N 3. P. 593. doi 10.1021/acsphotonics.6b00930

[6] Berini P., De Leon I. // Nature Photonics. 2012. V. 6. P. 16-24. doi $10.1038 /$ nphoton.2011.285

[7] Verhagen E., Dionne J.A., Kuipers L., Polman A. // Nano Letters. 2008. V. 8. N 9. P. 2925. doi 10.1021/n1801781g

[8] Basov D.N., Timusk T. // Rev. Mod. Phys. 2005. V. 77. P. 721. doi 10.1103/RevModPhys.77.721

[9] Kaina N., Causier A., Bourlier Y., Fink M., Berthelot T., Lerosey G. // Scientific Rep. 2017. V. 7. 15105. doi 10.1038/s41598-017-15403-8

[10] Gad S.A., Boshta M., Moustafa A.M., Abo El-Soud A.M. // Solid State Sci. 2011. V. 13. N 1. P. 23. doi 10.1016/j.solidstatesciences.2010.09.022

[11] Smirnova D.A., Iorsh I.V., Shadrivov I.V., Kivshar Yu.S. // JETP Letters. 2014. V. 99. N 8. P. 527. doi 10.1134/S002136401408013X

[12] Hajaj E., Shtempluk O., Kochetkov V., Razin A., Yaish Y. // Phys. Rev. B. 2013. V. 88. N 4. P. 045128. doi 10.1103/PhysRevB.88.045128

[13] Evseev D.A., Eliseeva S.V., Sementsov D.I. // Eur. Phys. J. Appl. Phys. 2017. V. 80. P. 10501. doi 10.1051/epjap/2017170167

[14] Евсеев Д.А., Семенцов Д.И. // Опт. и спектр. 2018. Т. 18. № 2. C. 235. doi 10.21883/OS.2018.02.45530.129-17

[15] Wang L., Yan Q., Xu H., Wang H., Zhang G., Zhou X. // Phys. Lett. A. 2016. V. 380. N 40. P. 3297. doi 10.1016/j.physleta.2016.07.063

[16] Gorbach A., Marini A., Alkorre H., Skryabin D. // Opt. Lett. 2013. V. 38. N 24. P. 5244. doi 10.1364/OL.38.005244

[17] Shkerdin G., Guo-Qiang H., Alkorre H., Stiens J. // J. Opt. 2017. V. 19. N . P. 015606. doi 10.1088/2040-8986/19/1/015606

[18] Xu W., Zhu Z., Liu K., Zhang J., Yuan X., Lu Q., Qin S. // Optics Express. 2015. V. 23. N 4. P. 5147. doi 10.1364/OE.23.005147

[19] Gan C.H., Chu H.S., Li E.P. // Phys. Rev. B. 2012. V. 85. P. 125431. doi 10.1103/PhysRevB.85.125431

[20] Фальковский Л.А. // УФН. 2012. Т. 182. № 11. С. 1223; Falkovsky L.A. // Phys. Usp. 2012. V. 55. P. 1140. doi 10.3367/UFNe.0182.201211i.1223
[21] Hanson G.W. // J. Appl. Phys. 2008. V. 103. P. 064302. doi 10.1063/1.2891452

[22] Novoselov K.S., Geim A.K., Morozov S.V., Jiang D., Zhang Y., Dubonos S.V., Grigorieva I.V., Firsov A.A. // Science. 2004. V. 306. P. 666-669. doi 10.1126/science.1102896

[23] Алисултанов 3.3., Мейланов Р.П. // ФТП. 2014. Т. 48. № 7. C. 951; Alisultanov Z.Z., Meilanov R.P. // Semiconductors. 2014. V. 48. N 7. P. 924. doi 10.1134/S1063782614070021

[24] Содха М.С., Гхатак А.К. Неоднородные оптические волноводы. М.: Связь, 1980. 216 с.; Sodha M.S., Ghatak A.K. Inhomogeneous Optical Waveguides. NY: Plenum Press, 1977. $269 \mathrm{p}$. 